\title{
Evaluation of a dialectical behavior therapy-informed partial hospital program: outcome data and exploratory analyses
}

\author{
John E. Lothes II, ${ }^{1}$ Kirk D. Mochrie, ${ }^{2}$ Emalee J.W. Quickel, ${ }^{3}$ Jane St. John ${ }^{4}$ \\ ${ }^{1}$ Department of Psychology, University of North Carolina Wilmington, Wilmington, NC; ${ }^{2}$ Psychology \\ Department, East Carolina University, Greenville, NC; ${ }^{3}$ Psychology Department, Loyola University Maryland, Baltimore, MD; \\ ${ }^{4}$ Delta Behavioral Health, Wilmington, NC, USA
}

\begin{abstract}
The use of dialectical behavioral therapy (DBT) among a variety of programs and patients has recently exploded. Of particular interest is the use of DBT in partial hospital (PH) programs due to the high number of severely ill and suicidal patients who participate in these programs. Recently, Lothes, Mochrie and St. John (2014) examined data from a local DBT-informed PH program and found significant reductions in depression, anxiety, hopelessness, and degree of suffering from intake to discharge. The present study examined these same four symptom constructs by assessing intake and discharge data for additional individuals enrolled in this DBT-informed PH program. In addition, lengths of stay and acuity ratings were analyzed to explore the relationship between these variables and symptom constructs. Significant symptom reduction in depression, anxiety, hopelessness, and degree of suffering from intake to discharge was found among high and medium acuity patients, replicating the results of Lothes et al. (2014). Further, individuals with the highest acuity saw the largest reduction in hopelessness symptoms the longer they participated in the program (i.e., a significant interaction effect between acuity and length of stay). This is meaningful given the connection between hopelessness and suicidal ideation/action, which is of particular concern for those charged with treating clinical populations. DBT-informed PH programs may be a cost-effective and useful way to treat high-risk patients who come from inpatient facilities. Future studies may wish to create follow-up periods (i.e., 3 months, 6 months) post-discharge to assess if symptom reduction remains.
\end{abstract}

Key words: Dialectical behavior therapy; Partial hospital; Symptom reduction.

Correspondence: John E. Lothes II, Department of Psychology, University of North Carolina Wilmington, 601 S. College Rd., Wilmington, NC 28403, USA.

Tel: +1.910.962.3370 - Fax: +1.910.962.7010.

E-mail: lothesj@uncw.edu

Contributions: KDM conducted literature searches and provided summaries of previous research studies as well as helped to design the study. JLII and JSJ were the primary data collectors and wrote the protocol. EJWQ significantly contributed to analyzing the data, writing the results section, and editing the manuscript. All authors have contributed to and have approved the final manuscript.

Conflict of interest: the authors declare no potential conflict of interest.

Citation: Lothes, J.E.II, Mochrie, K.D., Quickel, E.J.W., \& St. John, J. (2016). Evaluation of a dialectical behavior therapy-informed partial hospital program: outcome data and exploratory analyses. Research in Psychotherapy: Psychopathology, Process and Outcome, 19(2), 150-156. doi: 10.4081/ripppo.2016.219

Received for publication: 13 June 2016.

Accepted for publication: 5 October 2016.

This work is licensed under a Creative Commons Attribution NonCommercial 4.0 License (CC BY-NC 4.0).

(C) Copyright J.E. Lothes II et al., 2016

Licensee PAGEPress, Italy

Research in Psychotherapy:

Psychopathology, Process and Outcome 2016; 19:150-156

doi:10.4081/ripppo.2016.219

\section{Introduction}

There is growing literature examining the use of dialectical behavior therapy (DBT; Linehan, 1993a) in numerous settings with a variety of patients (Panos, Jackson, Hasan, \& Panos, 2013). DBT was originally developed as a one-year outpatient psychosocial treatment for individuals with borderline personality disorder (BPD; American Psychiatric Association, 2000; Simpson et al., 1998). Official DBT programs include four main components: skills group, individual therapy, crisis coaching and DBT consultation team. The skills group is where patients work through the four modules of DBT (i.e., core mindfulness, distress tolerance, emotion regulation, and interpersonal effectiveness) and are given handouts and worksheets for structured homework of practicing skills among group sessions (Linehan, 2014). These groups typically last 1.5 to 2.5 hours per week and are led by a facilitator and cofacilitator. In addition, patients receive individual therapy from a DBT-trained therapist who, among other responsibilities, reinforces skills learned in group through application to personal situations as identified using self-monitoring forms (i.e., diary/skills cards) the patient brings to each session (Linehan, 1993b).

Another component of DBT, a 24-hr crisis coaching phone, can be accessed at any time the patient needs coaching or help in a crisis situation to avoid engaging in 
the ineffective behaviors that resulted in referral to DBT in the first place. The remaining component, the DBT consultation team, sustains therapists in weekly meetings facilitating on-going personal practice of DBT by therapists, providing a group mindfulness practice, supporting fidelity to the DBT model, and assisting therapists' effectiveness via discussions of therapist/patient treatment interfering behaviors. Medical treatment is arranged as needed but is not an identified component of DBT. Fully implemented DBT programs have been shown to be effective in reducing suicidal behavior and emotion dysregulation for individuals with BPD (Linehan, 2014).

Interestingly, more recently DBT has been used to treat a wide variety of disorders (Koerner, 2012) over varying lengths of time (Lothes, Mochrie, \& St. John, 2014). Of particular interest is the use of DBT in partial hospital (PH) programs, which are used to help stabilize patients after a brief inpatient stay, or as an alternative to inpatient hospitalization (Neuhaus, 2006). PH programs allow patients with high-risk behaviors, such as suicide attempts, to transfer to a lower level of care after hospitalization. High-risk patients typically include those who experience specific mental health disorders, such as BPD, which are often accompanied by symptoms such as suicidal ideation (Pompili, 2012).

The $\mathrm{PH}$ program model can be especially effective in treating high-risk populations if effective treatment approaches are utilized. While there is a certain level of acuity in terms of medical necessity (as defined by the third party payers of each patient) that needs to be met to qualify for enrollment in a PH program, very little research has examined if acuity plays a role in how programs lead to symptoms reduction in patients. Although not specifically identified as a component of $\mathrm{PH}$ programs, one rationale for these programs is to save money by reducing future hospitalizations. In addition, $\mathrm{PH}$ programs can be an opportunity for teaching patients coping skills that they can utilize before stepping down to an intensive outpatient program or to individual therapy, and these skills can contribute to sustained stability at discharge, a strong strategy with the potential to contribute to lowering inpatient recidivism rates.

DBT may be particularly suited to PH programs due to the high-risk patients that are typically treated in this setting. Unfortunately, individuals with high-risk behaviors have been poorly treated in the past; however, with the emergence of DBT, therapists are better able to help reduce these symptoms (Panos et al., 2013). DBT in a PH setting requires an obviously modified schedule of skills delivery, and a shortened duration of individual therapy; however, all components of DBT are incorporated. DBT-informed $\mathrm{PH}$ programs have provided preliminary evidence in reducing symptoms of anxiety and depression from intake to discharge (Lothes et al., 2014); however, further research is needed to confirm treatment outcome data. $\mathrm{PH}$ programs are increasingly being required to measure treatment effec- tiveness and patient outcomes for insurance coverage purposes (Granello, Granello, \& Lee, 1999; Bateman \& Fonagy, 2003). Of particular concern is the time needed for patients to be involved in a $\mathrm{PH}$ program (e.g. length of stay) before significant symptom reduction can be found. Interestingly, Bateman and Fonagy (2003) found that individuals who complete long-term (several months to 18 months) $\mathrm{PH}$ programs use less inpatient services and have a higher intensity and a longer duration of functional improvements than those who seek other treatment or do not receive any treatment. More recently, Lothes et al. (2014) conducted a study assessing symptom reports from intake to discharge with a sample of 38 patients enrolled in a DBT-informed $\mathrm{PH}$ program. Results showed significant reductions from intake to discharge in depression, anxiety, hopelessness, and degree of suffering. Using DBT in PH program settings can be effective in reducing symptoms of various mental health disorders. To the best of our knowledge, no known research exists that examines variables like length of stay in the context of DBT-informed PH programs. In addition to overall efficacy of these program models, indicators such as length of stay that have an impact on funding and program evaluation considerations must be examined concurrently. While there is a certain level of acuity in terms of medical necessity that needs to be met to qualify for enrollment into a partial hospital program, very little research has examined if acuity plays a role in symptom reduction.

Further assessment of outcome data in DBT-informed $\mathrm{PH}$ programs is needed due to the relatively novel investigation of this topic. For example, the question of what length of time is needed to achieve symptom reduction in this population still rears its head. In addition, very little current research has been conducted assessing acuity ratings in this population. Examining acuity could provide useful data as to which patients are more likely to have quicker symptom reduction and progress through the program at a faster rate, thus allowing program directors to make better recommendations to insurance companies about the optimal program length of stay based on individualized patient needs; alternatively, allowing patients to stay in the program long enough for true change to occur may prevent relapse and save money on future hospitalizations and treatment.

Given these unknowns in the literature, the current study has two specific aims. First, the present study aims to replicate the previous study by Lothes et al. (2014) by measuring levels of reported depression, anxiety, hopelessness and degree of suffering at intake and discharge to assess the effectiveness of a DBT-informed PH program in the southeast region of the United States. The present study included individuals with broader diagnoses (e.g., eating disorders, substance dependence, psychotic disorders) to increase generalizability. In addition, the sample size was substantially larger providing sufficiently more power for more in depth statistical analyses. Second, the present study aims to include analyses that consider 
the impact of patient acuity and length of stay on symptom reduction. Understanding how these variables relate to patient outcomes may inform decisions made regarding treatment design and implementation, as well as policy decisions related to funding and relapse prevention.

The $\mathrm{PH}$ program is the same program evaluated in the first study by Lothes et al. (2014), which is an intensive day treatment option for adults. Patients meet 5 days per week, for 4 hours each day. During this time they are engaged in a curriculum based on Linehan's (1993b) DBT manual with in-depth skills training. The program has recently begun to implement Linehan's (2014) new DBT Skills Training Manual into practice; however, the participants for this analysis were still being taught with the previous manual. Treatment includes skills training in mindfulness, emotion regulation, distress tolerance, and interpersonal effectiveness, a morning check in process group, individual therapy, 24-hour coaching phone consultations, DBT team staff meetings, medication management, and psychiatric care. All individuals running the DBT treatment team have attended intensive Behavioral Tech DBT trainings and interns who conducted skills training were required to do a semester practicum of clinical training on DBT at the program facility. The skills modules were rotated through each week so that participants would be able to receive all four modules over the course of six weeks. The present study attempts to assess a number of constructs by examining intake and discharge data based on length of stay and acuity ratings for individuals enrolled in this DBT-informed PH program.

First, we hypothesized that the results would replicate those of the first study by Lothes et al. (2014) by showing significant decreases in depression, anxiety, hopelessness, and degree of suffering scores from intake to discharge; to extend this analysis, we were also interested in examining the whether the program would be effective at reducing symptoms for individuals with differing levels of acuity at intake. Second, it was hypothesized that acuity and length of stay in the program would have an interaction effect on symptom reduction. Specifically, individuals with higher acuity ratings entering treatment that also stayed longer in the program would have the greatest degree of symptom reduction.

\section{Materials and Methods}

\section{Participants}

Participants included 113 adults, ages 18-73 [mean $(M)=38.05$, standard deviation $(S D)=12.90]$. The sample was predominantly female $(\mathrm{N}=84,74.34 \%)$ and Caucasian $(\mathrm{N}=94,84.07 \%)$, and was comprised of patients who were evaluated for medical necessity for needing a higher level of care than an intensive outpatient program or continued individual outpatient therapy at intake and at discharge. Participants were diagnosed based on a clin- ical interview, assessment measures [i.e., Beck depression inventory II (BDI-II), Beck anxiety inventory (BAI), Beck hopelessness scale (BHS)] and review of medical records. Table 1 presents demographic characteristics, including diagnoses assigned according to DSM-IV-TR criteria (American Psychiatric Association, 2000). Participants did not receive compensation for participation.

\section{Depression}

The BDI-II (Beck, Ward, Mendelson, Moch, \& Erbaugh, 1961) was used to assess self-report ratings of depression at intake and discharge. The BDI-II is a 21-item measure; items contain a list of statements and the participant selects the statement that best describes them. For each item, statements range from those that indicate no depressive symptoms (i.e., I do not feel sad), which would receive a code of 0 , to those that indicate severe depressive symptoms (i.e., I am so sad and unhappy that I can't stand $i t$ ), which would receive a code of 3 . The BDI ranges from 0-13 (minimal), 14-19 (mild), 20-28 (moderate) and $29+$ (severe). The BDI is reliable and valid for use in general adult populations (Richter, Werner, Heerlein, Kraus, \& Sauer, 1998), as well as psychiatric populations (Beck, 1988; Beck, Steer, \& Garbin, 1988).

\section{Anxiety}

The Burns anxiety inventory (BAI; Burns, 1999) was used to assess self-report ratings of anxiety at intake and discharge. The $\mathrm{BAI}$ is a 33-item measure; answer choices for each item range from 0 (Not at all) to 3 ( $A$ lot). The BAI ranges from 0-20 (mild), through 21-30 (moderate), to $31+$ (severe).

Table 1. Demographic information $(n=113)$.

\begin{tabular}{llrr}
\hline & & M & SD \\
\hline Age (years) & & 38.05 & 12.90 \\
\hline \multirow{2}{*}{ Gender } & & N & $\mathbf{\%}$ \\
\hline Race & Male & 29 & 25.66 \\
& Female & 84 & 74.34 \\
\hline & Caucasian & 95 & 84.07 \\
& African American & 14 & 12.39 \\
& Hispanic & 2 & 1.77 \\
& Native American & 1 & 0.88 \\
& Other & 1 & 0.88 \\
\hline \multirow{2}{*}{ DSM-IV-TR diagnosis } & & & \\
& Major depressive disorder & 67 & 59.29 \\
& Bipolar disorders & 35 & 30.97 \\
& Anxiety disorders & 3 & 2.66 \\
& Substance dependence & 3 & 2.66 \\
& Psychotic disorders & 3 & 2.66 \\
& Eating disorders & 2 & 1.77 \\
\hline
\end{tabular}

M, mean; SD, standard deviation; DSM-IV-TR, diagnostic and statistical manual of mental disorders-fourth edition-TR. 


\section{Hopelessness}

The Beck hopelessness scale (BHS; Beck, 1988) was used to assess self-report levels of hopelessness at intake and discharge and is considered reliable and valid (Owen, 1992). The BHS is a 20 -item truelfalse measure; items include statements regarding attitudes about the future (i.e., I look forward to the future with hope and enthusiasm). The BHS ranges from 0-3 (minimal), through 4-8 (mild), 9-14 (moderate), to $15+$ (severe).

\section{Suffering}

Self-report degrees of suffering scores were collected on a one-item Likert scale ranging from 0 (No suffering at all) to 10 (Worst suffering ever) at intake and discharge.

\section{Acuity}

Participants were also given an acuity rating for overall impairment at intake based on their scores on the BDIII, BAI, and BHS by assigning points from all three measures according to each scale's criteria of low (scoring minimal or mild on BDI, BAI and BHS scales), moderate (scoring moderate on BDI, BAI and BHS scales), and high ratings (scoring severe on BDI, BAI, and BHS scales). If someone scored in the minimal range they received a 1 , in the moderate range they received a 2 , and in the severe range they received a 3 . This produced both a continuous (i.e., ranging from 3-9) and a categorical (i.e., low, medium or high) rating of patient acuity.

\section{Length of stay}

Length of stay was measured by total number of days in the program, consistent with previous research (Jiménez, Lam, Marot, \& Delgado, 2004).

\section{Procedure}

All patients who came into the $\mathrm{PH}$ program were asked to complete the BDI-II, BAI, BHS and reported their degree of suffering as part of the initial intake process. These measures were used as part of the intake process to assess for medical necessity into the $\mathrm{PH}$ program. Patients who did not meet medical necessity for the $\mathrm{PH}$ program were referred to the appropriate source; often an individual therapist or other appropriate community resources. Information on referrals was not collected. Patients also filled out the BDI-II, BAI, BHS, and self-reported degree of suffering at discharge. This information was used initially to help re-assess for medical necessity or to help patients step-down to a more appropriate program, such as intensive outpatient therapy or to weekly DBT skills groups supplemented with individual therapy.

The researchers later examined this information as an exploratory study approved by the Institutional Review Board at the University of North Carolina, Wilmington, NC. Since this data was not originally collected as part of the research design, there was no data collected for patients that did not meet medical necessity for the $\mathrm{PH}$ program.

\section{Results}

Results replicated the first study conducted by Lothes et al. (2014). See Table 2 for average symptom raw scores at intake and discharge. Raw scores are displayed to provide the greatest degree of clinical utility to practitioners. At intake, average depression, anxiety, and hopelessness scores for this sample fell in the severe, severe, and moderate ranges, respectively; at discharge, average depression, anxiety, and hopelessness scores for this sample fell in the mild, moderate, and mild ranges, respectively. A series of paired samples $t$-tests demonstrated significant changes in symptom reports for all four dependent variables: depression $[t(112)=13.47, \mathrm{P}<.001,95 \%$ confidence interval $(\mathrm{CI})(15.65,21.04)]$; anxiety $[t(112)=11.00$, $\mathrm{P}<.001,95 \%$ CI $(19.21,27.65)] ;$ hopelessness $[t$ (112) $=11.85, \mathrm{P}<.001,95 \% \mathrm{CI}(5.13,7.19)]$; and suffering $[t(112)=12.91, \mathrm{P}<.001,95 \% \mathrm{CI}(2.98,4.06)]$ (Figure 1).

The next goal of the current research was to see if the program was differentially effective for patients at different acuity levels. The program was effective at decreasing all four symptoms categories in high and medium acuity patients. According to a series of paired samples $t$-tests conducted with all high acuity clients $(\mathrm{N}=82)$, depression scores significantly decreased by $22.62(\mathrm{SD}=13.56)$ points on average $[t(81)=15.10, \mathrm{P}<.001,95 \%$ CI $(19.64$, 25.60)]; anxiety scores significantly decreased by 28.27 $(\mathrm{SD}=22.21)$ points on average $[t(81)=11.46, \mathrm{P}<.001$, $95 \%$ CI $(22.60,32.11)]$; hopelessness scores significantly decreased by $7.61(\mathrm{SD}=5.27)$ points on average $[t$ $(81)=13.09, \mathrm{P}<.001,95 \% \mathrm{CI}(6.45,8.77)]$; and suffering scores significantly decreased by $3.87(\mathrm{SD}=2.84)$ points on average $[t(81)=12.31, \mathrm{P}<.001,95 \% \mathrm{CI}(3.24,4.49)]$.

Table 2. Descriptive statistics $(n=113)$.

\begin{tabular}{lrc}
\hline & M & SD \\
\hline Acuity & 7.39 & 1.73 \\
\hline Days in program & 22.35 & 13.13 \\
\hline Depression intake & 33.00 & 12.99 \\
\hline Depression discharge & 14.65 & 12.42 \\
\hline Anxiety intake & 49.69 & 22.17 \\
\hline Anxiety discharge & 26.26 & 21.23 \\
\hline Hopelessness intake & 11.15 & 5.75 \\
\hline Hopelessness discharge & 4.99 & 5.00 \\
\hline Suffering intake & 6.53 & 2.31 \\
\hline Suffering discharge & 3.01 & 2.69 \\
\hline $\begin{array}{l}\text { M, mean; SD, standard deviation. Ms and SDs reflect raw scores on associated symptom } \\
\text { measures. }\end{array}$ & & \\
\end{tabular}


According to a series of paired samples $t$-tests conducted with all medium acuity clients $(\mathrm{N}=29)$, depression scores significantly decreased by $6.97(\mathrm{SD}=10.36)$ points on average $[t(28)=3.62, \mathrm{P}=.001,95 \% \mathrm{CI}(3.02,10.91)]$; anxiety scores significantly decreased by 13.86 $(\mathrm{SD}=22.90)$ points on average $[t(28)=3.26, \mathrm{P}=.003,95 \%$ CI $(5.15,22.57)]$; hopelessness scores significantly decreased by $2.28(\mathrm{SD}=4.38)$ points on average $[t(28)=2.80$, $\mathrm{P}=.009,95 \% \mathrm{CI}(0.61,3.94)]$; and suffering scores significantly decreased by $2.59(\mathrm{SD}=2.88)$ points on average $[t(28)=4.83, \mathrm{P}<.001,95 \%$ CI $(1.49,3.68)]$. There were only two clients that started the program in the low acuity category, so their data was unable to be statistically analyzed separately. However, they showed clinically meaningful decreases in all four-symptom areas as well. Their depression scores changed from an average of 10.00 to 2.00 , anxiety scores changed from an average of 6.00 to 4.50 , hopelessness scores changed from an average of 4.00 to 1.00 , and suffering scores changed from an average of 3.00 to 0.00 . However, these changes should be interpreted with caution.

To assess the possibility that length of stay might interact with patient acuity to produce symptom change, a series of moderated hierarchical linear regression analyses were conducted. Length of stay and patient acuity at intake were entered in step 1, and the interaction between length of stay and acuity in step two. The results showed that there was no interaction effect between acuity ratings at the start of the program and length of time spent in the program on depression, anxiety, or suffering score change. However, a moderated, hierarchical linear regression analysis showed that there was a significant interaction between acuity at the start of the program and length of time spent in the program on hopelessness score change $[F(3,109)=15.74$, $\mathrm{P}<.001$; Figure 2]. For ease of interpretation, acuity and length of stay are depicted as \pm 1 SD from the mean in Figure 2; continuous measures of these variables were entered into the regression analyses. The final model (predictors: acuity, length of stay, interaction term) explained $30.2 \%$ of the variability in hopelessness change. The addition of the interaction term explained an additional $4.20 \%$ of the variability in hopelessness change $(\mathrm{P}=.012)$.

\section{Discussion}

The purpose of the current study was to replicate and add to the findings of Lothes et al. (2014) by assessing the effectiveness of a DBT-informed PH program on depression, anxiety, hopelessness and degree of suffering based on patient length of stay and acuity ratings at intake. The present study results were able to successfully replicate Lothes et al. (2014) by showing significant decreases in depression, anxiety, hopelessness, and degree of suffering from intake to discharge for all participants. Thus, integrating DBT into PH programs' treatment protocol appears to be an effective method at decreasing symptoms that are associated with a wide variety of diagnoses and clinical concerns, including suicidal ideation and action.

Additionally, it was found that length of stay in the program moderated the relationship between acuity and change in hopelessness such that those who were most acute and stayed in the program for the longest had the greatest reduction in perceived hopelessness. This suggests that severely acute patients may benefit from staying longer in a PH program than less acute patients. In addition, preemptively assessing for hopelessness levels could prove more useful than anxiety or depression scores in determining length of stay needed in the program. Thus, a one-size-fits-all conceptualization of treatment in $\mathrm{PH}$ programs that is currently being practiced by insurance companies warrants some consideration. Perhaps some patients need more time in a $\mathrm{PH}$ program to receive the benefits of treatment. In addition, researchers have noted that significant declines in depression, anxiety, and hopelessness are important factors in the reduction of suicide

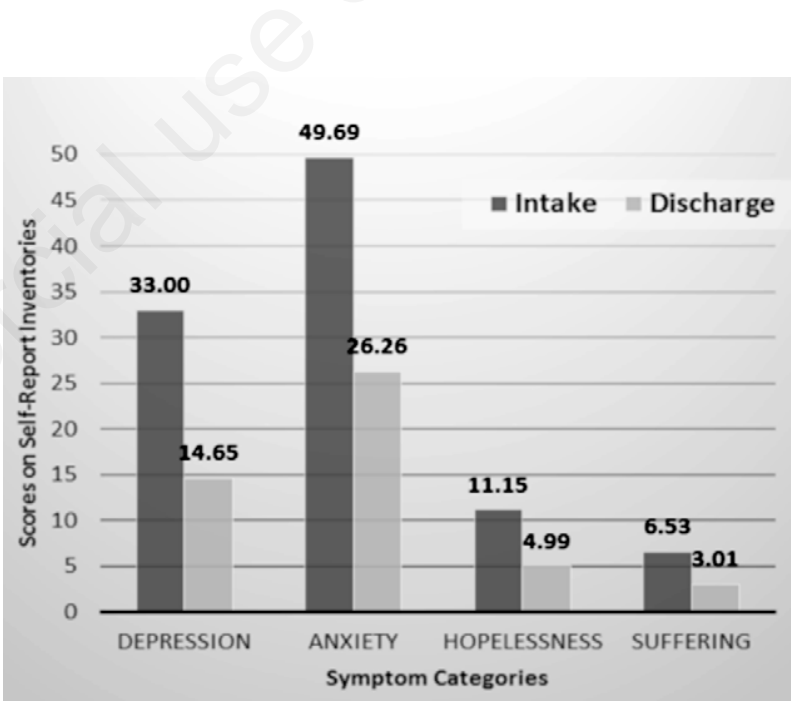

Figure 1. Change in hopelessness scores by acuity level and length of stay. 
risk, suggesting that a longer length of stay in a $\mathrm{PH}$ program may reduce the risk for suicide and the risk of return to an inpatient facility among this population (Neuhaus, 2006; Pompili et al., 2012); however, further studies are needed to specifically assess this.

The results of this study replicated and extended those of Lothes et al. (2014), suggesting that patients with higher levels of acuity may need longer lengths of stay to experience the same symptom reduction effect in a DBTinformed PH program as those with lower levels of acuity. The promising outcome is that there is still a significant decline in ratings of depression, anxiety, hopelessness and degrees of suffering from start to discharge, even for the most highly rated patients on these measures. Length of inpatient stay after exposure to DBT-informed treatment may be reduced and should be assessed in future studies. Economically, DBT could be a factor in looking at the reduction of overall treatment cost to the patient since attendance to a $\mathrm{PH}$ program is considerably more cost efficient than an inpatient stay (McMain et al., 2009). Batcheler (2005) reported on a pre-post study of DBT in New Zealand, finding a reduction in hospital days used (25.04 to 4 to 1.09 days) when comparing 12 months pretreatment, 12 months of DBT treatment, and 12 months post DBT treatment, respectively. Using a DBT-informed $\mathrm{PH}$ program as first choice instead of an inpatient facility may also be a cost savings method of treatment as well.

The study has several limitations. First, there is a lack of generalizability due to not having a control group. However, the researchers felt it would be ethically inappropriate to deprive high-risk patients of care by placing them on a control-waiting list. Also, the researchers attempted to reach out to other non-DBT PH programs to use as a comparison group. None of the clinics contacted were willing to let us collect data on their programs. In the future, comparison studies to other $\mathrm{PH}$ programs are recommended. Second, the measures used are all self-report and therefore may not have accurately assessed specific levels of psychological impairment at intake and discharge. Finally, data was not gathered on how many coaching phone calls were made by each patient, how many hospitalizations occurred while in program, or how many relapses of problem behaviors occurred (e.g., cutting, using, drinking, purging). Also not assessed was medication compliance.

\section{Conclusions}

Ideally, follow up studies (e.g., 3 months, 6 months and/or 12 months post discharge) are recommended for future studies to examine if depression, anxiety, hopelessness, and degree of suffering are still significantly below intake level. Further, it is suggested for follow up studies to examine rates of return to inpatient facilities. Finally, it may prove useful to examine differences in symptom reduction between more traditional cognitive behavioral therapy models of $\mathrm{PH}$ programs compared to DBT-informed $\mathrm{PH}$ programs.

\section{References}

American Psychiatric Association (2000). Diagnostic and statistical manual of mental disorders ( $4^{\text {th }} \mathrm{ed}$.). Washington, DC: American Psychiatric Association.

Batcheler, M. (2005). Dialectical behaviour therapy in New Zealand. Journal of the New Zealand College of Clinical Psychology, 15, 11-16.

Bateman, A., \& Fonagy, P. (2003). Health service utilization costs for borderline personality disorder patients treated with psychoanalytically oriented partial hospitalization versus general psychiatric care. American Journal of Psychiatry, 160, 169-171. doi:10.1176/appi.ajp.160.1.169

Beck, A.T. (1988). Beck hopelessness sale. New York, NY: The Psychological Corporation.

Beck, A.T., Steer, R.A., \& Garbin, M.G. (1988) Psychometric properties of the Beck depression inventory: twenty-five years of evaluation. Clinical Psychology Review, 8(1), 77-100.

Beck, A.T., Ward, C.H., Mendelson, M., Mock, J., \& Erbaugh, J. (1961). An inventory for measuring depression. Archives of General Psychiatry, 4, 561-571. doi:10.1001/archpsyc. 1961.01710120031004.

Burns, D.D. (1999). The feeling good handbook. New York, NY: William Morrow and Co.

Granello, D.H., Granello, P.F., \& Lee, F. (1999). Measuring treatment outcomes and client satisfaction in a partial hospitalization program. The Journal of Behavioral Health Services \& Research, 26, 50-63. doi: 10.1037/t00789-000.

Jiménez, R.E., Lam, R.M., Marot, M., \& Delgado, A. (2004). Observed-predicted length of stay for an acute psychiatric department, as an indicator of inpatient care inefficiencies. Retrospective case-series study. BMC Health Services Research, 4, 4. doi:10.1186/1472-6963-4-4.

Koerner K. (2012). Doing dialectical behavioral therapy: a practical guide. New York, NY: Guildford Press.

Linehan, M. (1993a). Cognitive-behavioral treatment of borderline personality disorder. New York, NY: Guilford Press.

Linehan, M. (1993b). Skills training manual for treating borderline personality disorder. New York, NY: Guilford Press.

Linehan, M. (2014). DBT skills training manual (2nd ed.). New York, NY: Guilford Press.

Lothes, J.E., Mochrie, K.D., \& St. John, J. (2014). The effects of a DBT informed partial hospital program on: depression, anxiety, hopelessness, and degree of suffering. Journal of Psychology and Psychotherapy, 4, 144. doi: 10.4172/21610487.1000144

McMain, S.F., Links, P.S., Gnam, W.H., Guimond, T., Cardish, R.J., Kormann, L., \& Streiner, D.L. (2009). A randomized trial of dialectical behavior therapy versus general psychiatric management for borderline personality disorder. American Journal of Psychiatry, 166, 1365-1374.

Neuhaus, E.C. (2006). Fixed values and a flexible partial hospital program model. President and Fellows of Harvard College, 14, 1-14. doi:10.1080/10673220500519706.

Owen, S.V. (1992). Review of the Beck hopelessness scale. In: J.J. Kramer \& J.C. Conoley (Eds.), The eleventh mental measurements yearbook (pp. 82-83). Lincoln, NE: University of Nebraska Press. 
Panos, P.T., Jackson, J.W., Hasan, O., \& Panos, A. (2013). Metaanalysis and systematic review assessing the efficacy of Dialectical Behavior Therapy (DBT). Research on Social Work Practice, 24(2), 213-223. doi:10.1177/1049731513503047.

Pompili, M. (2012), Exploring the phenomenology of suicide. Suicide and Life-Threatening Behavior, 40(3), 234-244. doi: 10.1521/suli.2010.40.3.234

Richter, P., Werner, J., Heerlein, A., Kraus, A., \& Sauer, H.
(1998). On the validity of the Beck depression inventory: a review. Psychopathology, 31, 160-168. doi:10.1159/000 066239.

Simpson, E.B., Pistorello, J., Begin, A., Costell, E., Levinson, J., Mulberry, S., ..., Stevens, M. (1998). Focus on women: use of dialectical behavior therapy in a partial hospitalization program for women with borderline personality disorder. Psychiatric Services, 49, 669-673. 